Nuclear Physics B128 (1977) 421-441

(c) North-Holland Publishing Company

\title{
ON THE BARE POMERON GRAPH IN TWO-DIMENSIONAL QCD *
}

\author{
M.B. EINHORN
}

Department of Physics, University of Michigan, Ann Arbor, Michigan 48109

\section{E. RABINOVICI}

Lawrence Berkeley Laboratory, University of California, Berkeley, California 94720

Received 12 April 1977

We present a detailed study of the bare pomeron graph in two-dimensional QCD in the $1 / N_{\mathrm{c}}$ approximation. The absence of a new singularity unrelated to quark parameters is derived. The "cylinder" graph is shown to induce renormalization of the vertex and intercept associated with quark and ti-quark exchange in the vacuum channel.

\section{Introduction}

Quantum chromodynamics (QCD), the theory of colored quarks interacting via colored gauge fields, may be the fundamental theory of strong interactions. Some time ago, its asymptotic freedom at short distances was demonstrated [1]. Although there are qualitative affirmations of the theory in the pattern of scaling violations observed in deep inelastic lepton scattering, it remains to be demonstrated that the precise, quantitative predictions of the theory for the asymptotic behavior are correct [2]. More seriously from a theoretical point of view, it has not been proved that quarks and gluons are confined in color singlet bound states. Finally, it is not known whether the spectrum of such states actually agree with the observed hadron spectrum.

Considering the theory in two space-time dimensions, 't Hooft showed how the properties of its solution might be obtained [3]. In many ways, this two-dimensional model is trivial compared to the QCD in four dimensions. Since the model is superrenormalizable, its asymptotic freedom is demonstrable by power counting. Because there are no transverse dimensions, the dynamical degrees of freedom associated with gluons can be gauged away. And since the Coulomb potential is linear, confinement occurs already in lowest order.

Nevertheless, the model is not without interest, since it can be used to study

* This work was supported under the auspices of the Division of Physical Research of the US Energy Research and Development Administration. 
some prototypical of asymptotically free gauge theories with confinement [4]. Its structure resembles parton models [5], and it is interesting to inquire whether those properties of parton models which are not derivable from the short-distance structure of an asymptotically free field theory actually survive. In addition, one can gain some insight into the paradox of parton models which suppose that quarks interact weakly but are confined. Interactions with currents have been studied from this point of view $[4,6]$. It is also interesting to consider properties of meson scattering in the model, not only to see how quarks are confined, but also because of its resemblance to dual models [7,9]. In ref. [9], we have summarized some of the properties obtained so far. Elsewhere [10], we present details concerning Regge behavior. Here, we would like to elaborate on the absence of the bare pomeron $[8,9]$ and the present details concerning the breaking of exchange degeneracy via vertex and intercept renormalizations due to the twisted loop or cylinder graph [11].

A related, extremely interesting subject which we will not touch on here is the relation between this field theory and the string model of hadrons [12] *.

In the next section, we review qualitatively the results obtained previously and summarize results to be derived in this paper. In sect. 3 , we present the detailed justification of the results on the pomeron and Regge-like renormalizations. Sect. 4 concludes with a brief summary and suggestions for future investigations.

\section{Meson scattering in (QCD) 2}

Following 't Hooft [7], we perform an expansion of Feynman graphs in $1 / N_{c}$ for fixed $g^{2} N_{\mathrm{c}}$, where $g$ is the gauge coupling constant and $N_{\mathrm{c}}$ is the number of colors. To leading order, the theory is described by non-interacting, color-singlet, mesonic bound states of a quark-antiquark pair. In two dimensions, we may choose gauges (e.g. $A_{-}=0$ ) in which the gluon field has no self-coupling, but represents a Coulomb potential acting between quarks. The "radial" excitations in the potential produce a meson spectrum with masses $\mu_{n}^{2}(n=1,2, \ldots)$ which, for high excitations, behaves like a linear "trajectory"

$$
\mu_{n}^{2} \rightarrow \alpha^{\prime} n, \quad \text { as } n \rightarrow \infty \text {. }
$$

The scattering of these bound states appears first in order $1 / N_{\mathrm{c}}$. To this order, all the Feynman diagrams for two-body scattering are planar diagrams with no internal quark loops. These may be classified according to the three cyclicly inequivalent planar graphs as $(s t),(u t)$, or (su) diagrams, in a language familiar from the Veneziano model [14]. For example, all the graphs contributing to the $(u t)$ diagram are depicted in fig. 1. These graphs pictorially resemble the Harari-Rosner duality diagrams [3]; it will be seen that the resemblance is not just superficial. The full amplitude $A$ is

\footnotetext{
* For an intriguing marriage of $(\mathrm{QCD})_{2}$ with a string in four dimensions, see Tye [13].
} 


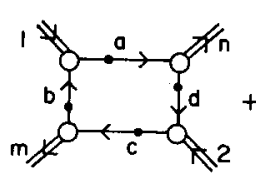

(a)

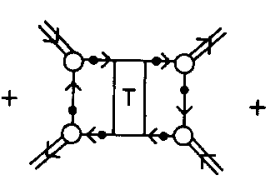

(b)

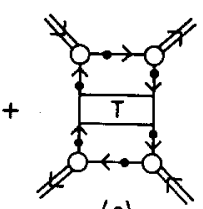

(c)

Fig. 1. The ( $u t$ ) amplitude to leading order in $1 / N$ in a gauge without self-coupled gluons (a) No quark rescattering, (b) $s$-channel rescattering and (c) $t$-channel rescattering.

the sum of $(s t),(u t)$, and (su) graphs. Its imaginary part is given by a sum over mesonic resonances in the $s$-channel. At the same time, the leading asymptotic behavior for forward scattering is power-behaved and given by

$$
A(s) \rightarrow\left((-s)^{\alpha} \text { bd }+(-u)^{\alpha} \text { bd }\right) \gamma_{1 n} \gamma_{2 m} .
$$

The parameter $\alpha_{b d}$ is additive in the exchanged quarks b, d (fig. 1) and is given by

$$
\alpha_{b d}=-\beta_{b}-\beta_{d},
$$

where $\beta$ is determined by the renormalized quark mass $m^{2}$ according to

$$
\pi \beta_{i} \cot \pi \beta_{i}=-m_{i}^{2}, \quad(i=\mathrm{b}, \mathrm{d}),
$$

This behavior reflects the fact, emphasized by Feynman [5], that mesons scatter by exchanging their wee constituents - in this case the slow moving valence quarks. The high energy behavior expressed by eq. (2) corresponds to what, in four dimensions, would be called Regge asymptotic behavior, governed by Regge-pole exchanges in the $t$-channel which factorize and are strongly exchange degenerate. We add that, from the view point of hadron unitarity, there are no quark discontinuities of the amplitude. Indeed, the quark discontinuities of fig. 1a are cancelled by similar contributions to figs. $1 \mathrm{~b}$ and $1 \mathrm{c}[10]$.

The lowest order contributions to the class of planar diagrams having two quark boundaries and no handles (usually identified with the "bare" pomeron $[7,14]$ appears in order $1 / N_{\mathrm{c}}^{2}$. (See fig. 2.) Trusting by now that the imaginary part will be given by a sum over mesonic intermediate states, we will calculate this by actually performing the sum:

$$
\operatorname{Im} P=\sum_{m, n}\left|A_{12 \rightarrow n m}(s)\right|^{2} \rho_{n m}(s) .
$$

Here $P$ denotes the bare pomeron amplitude; $A_{12 \rightarrow n m}(s)$, the ( $\left.u t\right)$ amplitude depicted in fig. 1 ; and $\rho_{n m}$, the phase-space factor proportional, in two dimensions, to $\lambda^{-1 / 2}\left(s, \mu_{n}^{2}, \mu_{m}^{2}\right)$, where $\lambda$ is the triangular function. Although the sum includes only two-body-intermediate states to this order in $1 / N_{\mathrm{c}}$, we must remember that the spectrum of states extends to infinity. Consequently, as $s$ increases, the number of channels open also increases. 


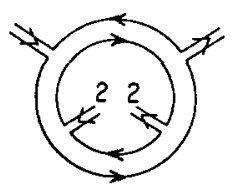

(a)

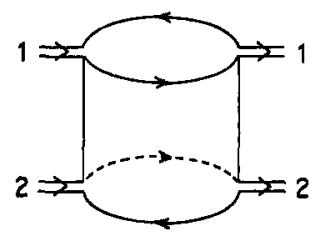

(b)

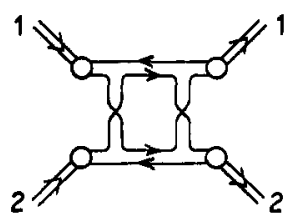

(c)

Fig. 2. Three equivalent representations of the bare pomeron graph: (a) planar graph with two quark boundaries, (b) cylinder or tube showing gluonic exchanges in the $t$-channel, (c) twisted loop displaying mesonic intermediate states. (The many $T$-matrix insertions possible have been suppressed.)

We are primarily intersted in the question of whether, by unitarity, the high energy of $\operatorname{Im} P$ is dominated by a new power whose exponent is independent of quark parameters. To gain some insight into how a "bare" pomeron might emerge, consider the following argument: there is a scaling amplitude to find a quark of momentum fraction $x_{1}\left(x_{2}\right)$ in meson $1(2)$. The quark from meson 1 binds with the anti-quark meson 2 to form meson $\mu_{n}$ with $\mu_{n}^{2}=x_{1}\left(1-x_{2}\right) s$. Similarly, $\mu_{m}^{2}$ $=x_{2}\left(1-x_{1}\right) s$. Suppose there were a scaling amplitude for this to occur,

$$
A_{12 \rightarrow n m}(s) \rightarrow A\left(x_{1}, x_{2}\right) \quad \text { as } s \rightarrow \infty \text {. }
$$

One can easily show that

$$
\sum_{n m} \rho_{n m} \approx \int \mathrm{d} \mu_{n}^{2} \mathrm{~d} \mu_{m}^{2} \rho_{n m}(s) \propto s \int \mathrm{d} x_{1} \mathrm{~d} x_{2}
$$

so

$$
\operatorname{Im} P \propto s \int \mathrm{d} x_{1} \mathrm{~d} x_{2}\left|A\left(x_{1}, x_{2}\right)\right|^{2} .
$$




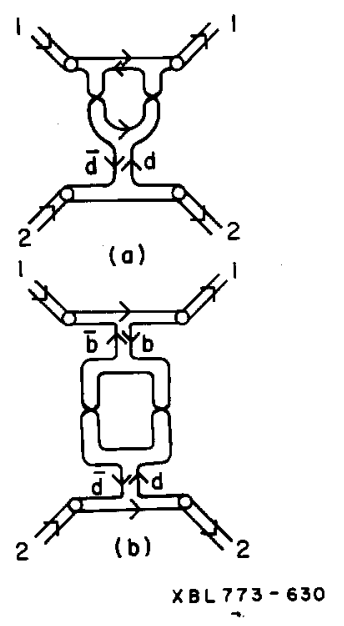

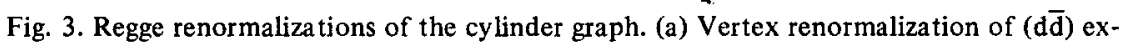
change. (b) Intercept renormalization: Mixing between $(b \bar{b})$ and $(d \bar{d})$ exchanges.

Thus, if $A_{12 \rightarrow n m}(s)$ scaled in this region of phase space, there would emerge from the unitarity sum a new power, unrelated to quark parameters, corresponding to intercept one! As we shall discuss in the next section, the result of the detailed calculation is that, although each time-ordered contribution scales as described above, terms conspire in pairs to cancel and we find that $\operatorname{Im} P$ receives a contribution from this region of phase space which vanishes at least as fast as $s^{-1}$.

It is also interesting to see whether exchange degeneracy is broken by renormalization of "vacuum" Regge exchanges by the cylinder graph. Contrary to the result on the pomeron, we find that other regions of phase space behave more or less as expected. The region where one intermediate mass, say $\mu_{m}$, becomes large but where the other mass, $\mu_{n}$, stays finite (or vice versa) leads to a vertex renormalization of the planar graph. (See fig. 3a.) The corresponding behavior of the amplitude $A_{12 \rightarrow n m}(s)$ is $\left(\mu_{m}^{2}\right)^{-\beta}$. This contribution leads to $\operatorname{Im} P \sim s^{-2 \beta}$ and so breaks strong exchange degeneracy of the "vacuum" exchanges.

Next we study the region of phase space in which both intermediate masses become large but such that $\rho=\mu_{n}^{2} \mu_{m}^{2} / s$ remains finite. Experience with the dual model leads us to expect this to give rise to renormalization of the intercepts in the vacuum channel. (See fig. 3b.) The corresponding asymptotic behavior of $A_{12 \rightarrow n m}(s)$ is $\left(\mu_{m}^{2}\right)^{-\beta_{\mathrm{b}}}\left(\mu_{n}^{2}\right)^{-\beta_{\mathrm{d}}}$. For $\beta_{\mathrm{b}} \neq \beta_{\mathrm{d}}$, this corresponds to mixing between the two trajectories. When $\beta_{\mathrm{a}}=\beta_{\mathrm{b}} \equiv \beta$ it gives $\operatorname{Im} P \sim s^{-2 \beta}$ ln $s$ which is, as usual, interpretable as the first term in an expansion of an intercept shift. Thus, weak exchange degeneracy is also broken in the vacuum channel. We turn now to the detailed calculation of all these effects. 


\section{Mathematical details}

This section consists of a detailed treatment of the scattering process; its outline is as follows. First, we will cast the full amplitude into a form amenable to investigation in all regions of phase space. The main result obtained is eq. (21). Next, the asymptotic energy dependence is studied in the various kinematic regions of physical interest, in particular, in the limit of Regge behavior and associated renormalizations and in the pomeron limit. In each case for illustrative purposes, we single out a particular term for which the detailed analysis is presented.

\subsection{Calculation of the (ut) amplitude}

Following 't Hooft [3], we work with light-cone coordinates, $p_{\mp}=\sqrt{\frac{1}{2}}\left(p^{0} \pm p^{1}\right)$, with metric tensor $g_{++}=g_{--}=0, g_{-+}=1$. In addition, we choose the light-cone gauge $A_{-}=0$. Because this gauge is not parity-invariant, the symmetry between right and left movers is destroyed. However, this is the only gauge in which the solution has been explicitly worked out. In this gauge, it turns out that all quantities studied depend ultimately on the (Lorentz-invariant) ratios of the minus components of the various momenta. These may then be evaluated in the center-of-mass frame where we have chosen:

$$
\begin{array}{ll}
p_{1}=\left(E_{1}, p\right), & p_{n}=\left(E_{n}, p^{\prime}\right), \\
p_{2}=\left(E_{2},-p\right), & p_{m}=\left(E_{m},-p^{\prime}\right) .
\end{array}
$$

We shall use notation and conventions employed earlier [15]. For those unfamiliar with this work, we summarize the basic results in an appendix. We begin with the exact calculation of the $(u t)$ planar diagram, fig. 1 , whose square gives $\operatorname{Im} P$. The first diagram, fig. 1a, has only free quarks as intermediate states. Whatever contribution they give to the $u$-channel discontinuity must be cancelled by other, similar contributions so that the imaginary part can be expressed solely in terms of mesonic intermediate states. This cancellation will be demonstrated shortly. The amplitude corresponding to fig. $1 \mathrm{a}$ is

$$
\begin{aligned}
& T_{\mathrm{A}}=-i\left(\sqrt{ } \frac{\pi}{N}\right)^{4} N 2^{4} \int \frac{\mathrm{d}^{2} k}{(2 \pi)^{2}} S\left(k-p_{1}\right) \Gamma_{1}\left(k, p_{1}\right) S(k) \Gamma_{n}\left(k, p_{n}\right) S\left(k-p_{n}\right) \\
& \quad \times \Gamma_{2}\left(k-q, p_{2}\right) S(k-q) \Gamma_{m}\left(k-q, p_{m}\right),
\end{aligned}
$$

where $q=p_{1}-p_{m}$ is the $u$-channel momentum. The amplitude for fig. $1 \mathrm{~b}$ is

$$
\begin{aligned}
T_{\mathrm{B}_{\mathrm{a}}} & =\left(\sqrt{ } \frac{\pi}{N}\right)^{4} N^{2} 2^{6} \int \frac{\mathrm{d}^{2} k}{(2 \pi)^{2}} \frac{\mathrm{d}^{2} l}{(2 \pi)^{2}} S(k-q) \Gamma_{m}\left(k-q, p_{m}\right) S\left(k-p_{1}\right) \Gamma_{1}\left(k, p_{1}\right) S(k) \\
& \times T\left(k_{-}, l_{-} ; q\right) S(l) \Gamma_{n}\left(l, p_{n}\right) S\left(l-p_{n}\right) \Gamma_{2}\left(l-q, p_{2}\right) S(l-q)
\end{aligned}
$$


The amplitude for fig. $1 \mathrm{c}$ is

$$
\begin{aligned}
T_{\mathrm{c}} & =\left(\sqrt{ } \frac{\pi}{N}\right)^{4} N^{2} 2^{6} \int \frac{\mathrm{d}^{2} k}{(2 \pi)^{2}} \frac{\mathrm{d}^{2} l}{(2 \pi)^{2}} S\left(k-p_{1}\right) \Gamma_{1}\left(k, p_{1}\right) S(k) \Gamma_{n}\left(k, p_{n}\right) S\left(k-p_{n}\right) \\
& \times T\left(k-p_{n}, l-p_{n} ; \Delta\right) S\left(l-p_{n}\right) \Gamma_{2}\left(l-q, p_{2}\right) S(l-q) \Gamma_{m}\left(l-l, p_{m}\right) S\left(l-p_{1}\right)
\end{aligned}
$$

where $\Delta=p_{1}-p_{n}$ is the $t$-channel momentum.

Since the only dependence on the plus components of the loop momenta enters via the propagators, whose exact form is known, the integration over these components may be explicitly performed. This brings the "covariant" amplitudes above into a sum of "time"-ordered diagrams as in old-fashioned perturbation theory. To illustrate, consider the plus-integration in the amplitude $T_{\mathrm{B}}$ :

$$
I_{\mathrm{B}}=\int \mathrm{d} k_{+} S(k-q) S\left(k-p_{1}\right) S(k) \int \mathrm{d} l_{+} S(l) S\left(l-p_{n}\right) S(l-q) .
$$

These integrations may be easily performed by contour integration yielding

$$
\begin{aligned}
I_{\mathrm{B}} & =\frac{i \pi}{\Delta E\left(p_{1}, k\right)} \frac{\theta\left(q_{-}<k_{-}<p_{1-}\right)}{\Delta E\left(p_{m}, k-q\right)}-\frac{\theta\left(0<k_{-}<q_{-}\right)}{\Delta E(q, k)} \\
& \times \frac{i \pi}{\Delta E\left(p_{n}, l\right)} \frac{\theta\left(q_{-}<l-<p_{n-}\right)}{\Delta E\left(p_{2}, l-q\right)}-\frac{\theta\left(0<l_{-}<q_{-}\right)}{\Delta E(q, l)},
\end{aligned}
$$

where the "energy denominator" in each case is the function

$$
\Delta E(r, s)=\frac{r^{2}}{r_{-}}-\frac{m^{2}}{r_{-}-s_{-}}-\frac{m^{2}}{s_{-}} .
$$

For notational simplicity, we have suppressed possible differences in the masses for quarks of different flavors. We shall restore this dependence in our final formulas. When this result, eq. (15) is inserted back into $T_{\mathrm{B}}$, we get a sum of four $x_{-}$ordered terms depicted in fig. 4. Similarly, one can perform the plus-integrations in $T_{\mathrm{A}}$ and $T_{\mathrm{c}}$ leading to diagrams depicted in fig. 5 and fig. 6 . (The names assigned the various time-ordered amplitudes in figs. 4-6 will be useful later.) The corresponding formulas are not very illuminating, depending as they do on energy denominators and proper vertices, both of which are gauge dependent. The energy denominators are also misleading, their vanishing represents free quark singularities which eventually must not occur. As discussed previously [15], these quark singularities are cancelled by zeros in the proper vertices through relations such as

$$
\Gamma_{i}(p ; r)=-\Delta E(r, p) \phi_{i}\left(p_{-} / r_{-}\right), \quad 0<p_{-} / r_{-}<1 .
$$

When $p_{-} / r_{-} \notin(0,1)$, we may use eq. (A.6) to write it in terms of an integral over 

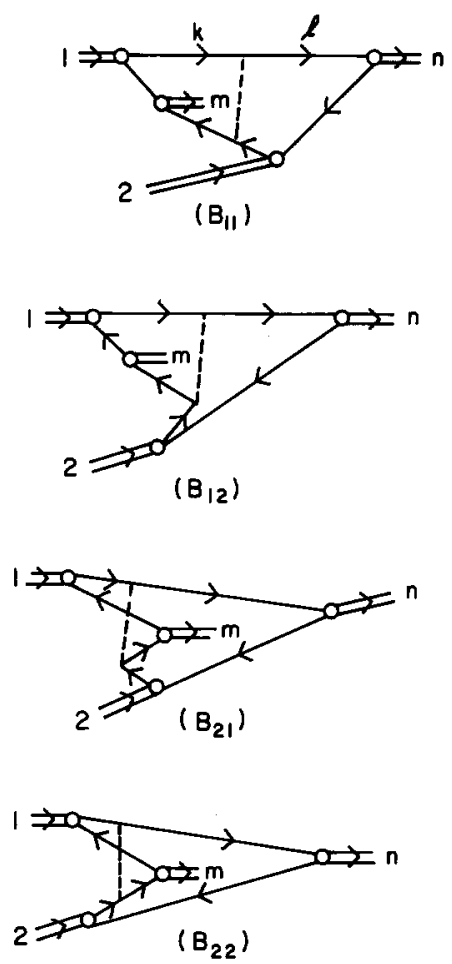

Fig. 4. "Old-fashioned" perturbation theory diagrams for $T_{\mathrm{B}}: B_{11}\left(0<k_{-}, l_{-}<q_{-}\right)$; $B_{12}\left(0<k_{-}<q_{-} ; q_{-}<l_{-}<p_{n_{-}}\right) ; B_{21}\left(q_{-}<k_{-}<p_{1-}, 0<l_{-}<q_{-}\right) ; B_{22}\left(q_{-}<k_{-}<p_{1-}\right.$, $q_{-}<l_{-}<p_{n}$ ) (The dashed line represents the quark-antiquark $T$-matrix; all quark propagators are understood to be fully dressed.)

the wave function $\phi_{i}$. Such formulas have the advantage of eliminating any gauge dependence, since the wave function $\phi_{i}(x)$ is gauge invariant [15]. In general, the $T$-matrix is also gauge-dependent, so it is useful to display the gauge dependence explicitly. For this purpose, it is useful to note eqs. (A.7) and (A.9). For $k_{-} / r_{-} \in(0,1)$ is is easy to show that

$$
\frac{N}{\pi} T\left(k_{-}, l_{-} ; r\right)=\Delta E(k, r) \frac{1}{r_{-}} \int_{0}^{1} \frac{\mathrm{d} v G\left(x, v ; r^{2}\right)}{(y-v)^{2}},
$$

where $x=k_{-} / r_{-}, y=l_{-} / r_{-}$. If, in addition, $y \in(0,1)$, one can show that

$$
\frac{N}{\pi} T\left(k_{-}, l_{-} ; r\right)=\Delta E(k, r) \delta\left(k_{-}-l_{-}\right)-\Delta E(k, r) \Delta E(l, r) G\left(x, y ; r^{2}\right) .
$$

The $\delta$-function arises because of the long-range force. In practice, it has the effect 


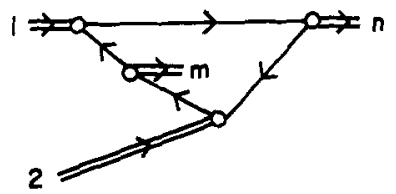

$\left(A_{1}\right)$
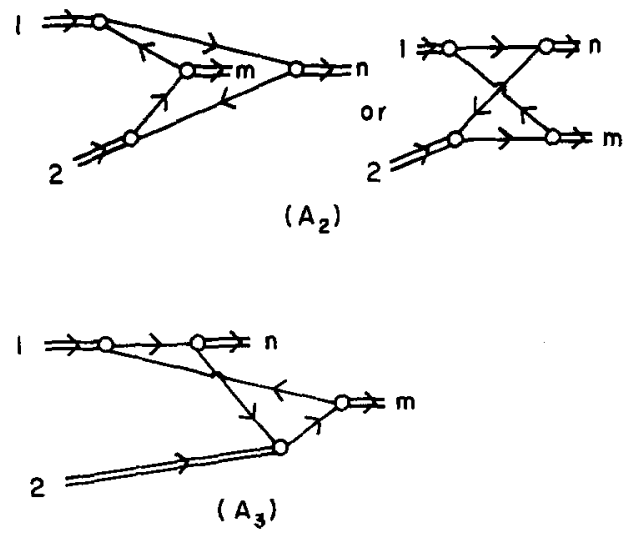

Fig. 5. "Old-fashioned" perturbation theory diagrams for $T_{\mathrm{A}}: A_{1}\left(0<k_{-}<q_{-}\right)$; $A_{2}\left(q_{-}<k_{-}<\phi_{n_{-}}\right) ; A_{3}\left(p_{n_{-}}<k_{-}<p_{1-}\right)$.

of cancelling con tributions from "disconnected" diagrams such as fig. 1a. For example, the $\delta$-function contribution to the amplitude corresponding to $B_{11}$ precisely cancels the corresponding time-ordered contribution from $T_{\mathrm{A}}$, namely $A_{1}$. Quark intermediate states are thereby eliminated, as expected in a theory of confinement. Indeed, one can show that $A_{2}$ is cancelled by the $\delta$-function pieces coming from $B_{22}$ plus $C_{11} ; A_{3}$, by $C_{22}$.

The second term in eq. (19) above supplies two additional inverse energy denominators necessary to cancel any quark singularities remaining. Combining all these operations leads to fairly compact expressions. For example, one can write

$$
\begin{aligned}
A_{1} & +B_{11}=\frac{4 \pi}{N} q_{-}^{2} p_{2-} p_{m-} \iiint \int_{0}^{1} \mathrm{~d} x \mathrm{~d} y \mathrm{~d} u \mathrm{~d} v\left[\frac{\phi_{m}(u) \phi_{1}\left(x q_{-} / p_{1-}\right)}{\left(u p_{m-}+(1-x) q_{-}\right)^{2}}\right. \\
& \left.\times G\left(x, y ; q^{2}\right) \frac{\phi_{n}\left(y q_{-} / p_{n-}\right) \phi_{2}(v)}{\left(u p_{2-}+(1-y) q_{-}\right)^{2}}\right] .
\end{aligned}
$$

Notice that this amplitude is purely real, as expected. All integrations run from 0 to 1. The amplitude is free of any quark singularities and is manifestly Lorentz invariant and gauge invariant. 

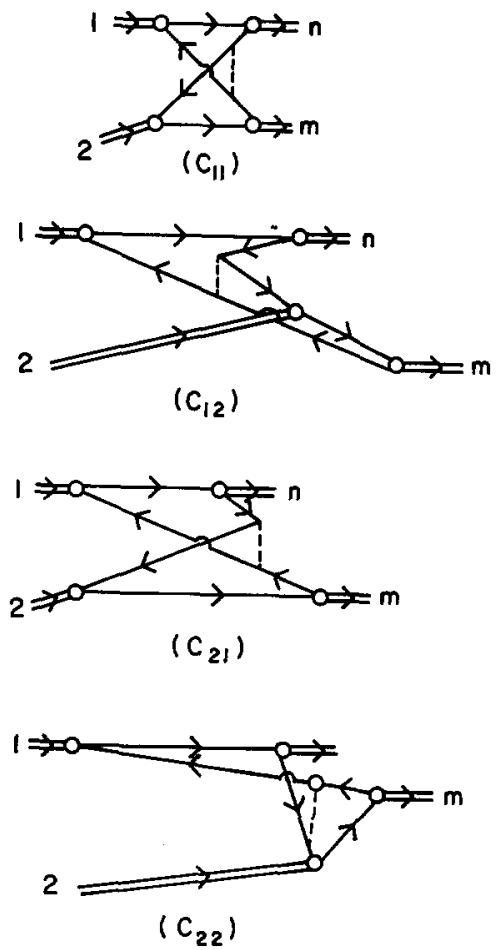

Fig. 6. "Old-fashioned" perturbation theory diagrams for $T_{\mathrm{c}}: C_{11}\left(0<k_{-}<p_{n-}, q_{-}<l_{-}<p_{n-}\right)$; $C_{12}\left(0<k_{-}<p_{n_{-}}, p_{n_{-}}<l_{-}<p_{1_{-}}\right) ; C_{21}\left(p_{n_{-}}<k_{-}<p_{1-}, q_{-}<l_{-}<p_{n_{-}}\right): C_{22}\left(p_{n_{-}}<k_{-}\right.$, $\left.<p_{1-}\right)$.

The same procedure, applied to all diagrams, when added together yields the complete result:

$$
\begin{aligned}
& \frac{N}{4 \pi} A_{12 \rightarrow n m}(s)=p_{2} p_{m} \iint \mathrm{d} u \mathrm{~d} v\left[\frac{\phi_{1}\left(\frac{q+v p_{2}}{p_{1}}\right)-\phi_{1}\left(\frac{q+u p_{m}}{p_{1}}\right)}{\left(v p_{2}-u p_{m}\right)^{2}}\right] \\
& \times \phi_{n}\left(\frac{q+v p_{n}}{p_{n}}\right) \phi_{2}(v) \phi_{m}(u)+p_{2} p_{n} \iint \mathrm{d} u \mathrm{~d} v \frac{\left[\phi_{1}\left(\frac{q+v p_{2}}{p_{1}}\right)-\phi_{1}\left(\frac{u p_{n}}{p_{1}}\right)\right]}{\left(p_{2}(1-v)-p_{n}(1-u)\right)^{2}} \\
& \times \phi_{n}(u) \phi_{2}(v) \phi_{m}\left(v p_{2} / p_{m}\right)+q^{2} p_{2} p_{m} \iiint \int \mathrm{d} x \mathrm{~d} y \mathrm{~d} u \mathrm{~d} v G(x, y ; U) \phi_{m}(u) \phi_{2}(v) \\
& \quad \times \frac{\left[\phi_{n}\left(\frac{y q}{p_{n}}\right)-\phi_{n}\left(\frac{q+p_{2} v}{p_{n}}\right)\right]\left[\phi_{1}\left(\frac{x q}{p_{1}}\right)-\phi_{1}\left(\frac{q+u p_{m}}{p_{1}}\right)\right]}{\left(p_{m} v+q(1-y)\right)^{2}} \frac{\left.p_{m} u(1-x)\right)^{2}}{\left(p_{2}\right.}
\end{aligned}
$$




$$
\begin{aligned}
& +\Delta^{2} p_{2} p_{n} \iiint \int \mathrm{d} x \mathrm{~d} y \mathrm{~d} u \mathrm{~d} v G(x, y ; T) \phi_{n}(u) \phi_{2}(v) \\
& \times \frac{\left[\phi_{m}\left(\frac{p_{2}+y \Delta}{p_{m}}\right)-\phi_{m}\left(\frac{v p_{2}}{p_{m}}\right)\right]}{\left(\Delta y+p_{2}(1-v)\right)^{2}} \frac{\left[\phi_{1}\left(\frac{p_{n}+x \Delta}{p_{1}}\right)-\phi_{1}\left(\frac{u p_{n}}{p_{1}}\right)\right]}{\left(\Delta x+p_{n}(1-u)\right)^{2}}
\end{aligned}
$$

All momenta appearing in the formula refer to their minus components, i.e., $q / p_{1}$ $\equiv q_{-} / p_{1-} ;$ all in tegrals run over $(0,1)$. Here $U \equiv q_{\mu}^{2}, T \equiv \Delta_{\mu}^{2}$.

The first term in the expression above comes from $T_{\mathrm{A}}$ and the Born term of $T_{\mathrm{B}}$; the second, from $T_{\mathrm{A}}$ and the Born term of $T_{\mathrm{c}}$. The third term comes from the rest of $T_{\mathrm{B}}$; the fourth, of $T_{\mathrm{c}}$. The diagrammatic interpretation of each of these terms is represented in fig. 7 . In order to avoid having quark and gluon lines crossing each other, we have drawn figs. 7 in a planar fashion. However, one should understand that, like figs. 4-6, they refer to particular $x_{-}$ordered contributions and that the Coulomb potential is "instantaneous" in $x_{-}$. No confusion can arise from this when one recalls that all quark and antiquark constituents have their minus components of momenta restricted so that the arguments of all mesonic wave function lie in the interval $(0,1)$. The relative minus signs between pairs of terms can be understood by observing that they differ by the coupling of a gluon to a quark or to an antiquark in a color singlet meson. The minus sign reflects the opposite color charge carried by the quark and the antiquark. Within the class of $A_{-}=0$ gauges, one can easily show that the piece of $T_{\mathrm{A}}$ contributing to the first term and the Born contribution of $T_{\mathrm{B}}$ are not separately gauge invariant. Only when combined as in the first term do they give a gauge-invariant quantity and, in so doing, remove the infrared singularity. (An analogous remark applies to the second term.) the third and fourth terms differ in this regard, since there is no infrared singularity within the region of integration. For example, each of the four contributions to the third term appears to be gauge invariant.

Looking at the diagrams corresponding to each piece of eq. (21), one can easily develop heuristic rules for writing down the form of the final answer directly. We will not pause here to discuss them but will pass on to the study of the asymptotic behavior of this amplitude. Note that in the case meson 1 is massless, the wave function $\phi_{1}(x)$ is constant for any $x$ leading thus by virtue of eq. (21) to a zero scattering amplitude. This demonstrates one aspect of the decoupling of zero-mass mesons conjectured by Callen et al. [4]. Proving the decoupling of a zero-mass left-moving meson (2) seems much more complicated in this gauge.

\subsection{Asymptotic behavior of the scattering amplitude}

In this section, we discuss the high energy behavior of the (ut) amplitude, eq. (21), in various kinemtical limits. We are especially interested in its contributions to Im P eq. (5), so we shall also need to know the behavior of the phase space factor. In table 1 , we summarize the values of the minus components of all the relevant momenta and the triangular function $\lambda\left(s, \mu_{n}^{2}, \mu_{m}^{2}\right)$. 

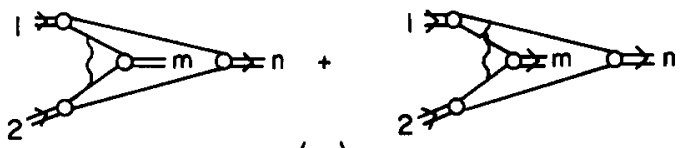

(a)
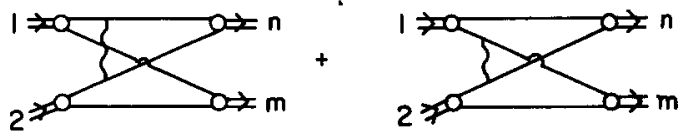

(b)
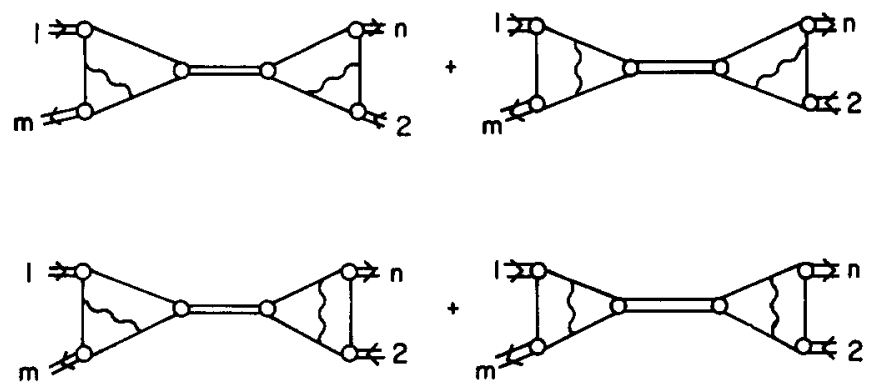

(c)
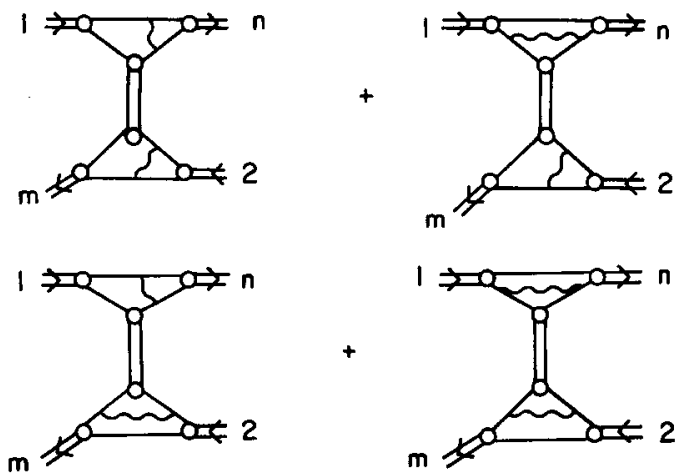

(d)

Fig. 7. Diagrammatic representation of each of the integrals in (ut) amplitude, eq. (21). (The wavy line denote gluon exchange. To avoid confusion because of lines crossing, we have not drawn (c) and (d) in "time"-ordered form even though the gluon exchange occurs instantaneously.)

\subsubsection{The "Regge" limit}

The high energy behavior of the (ut)-amplitude for fixed masses $\mu_{n}, \mu_{m}$ corresponds to a Regge limit and is expected to behave as $s^{\alpha}$ as $s \rightarrow \infty$. It turns out that every term in eq. (21) gives the same result, so we will illustrate the feature 
M.B. Einhorn, E.Rabinovici / Bare pomeron graph

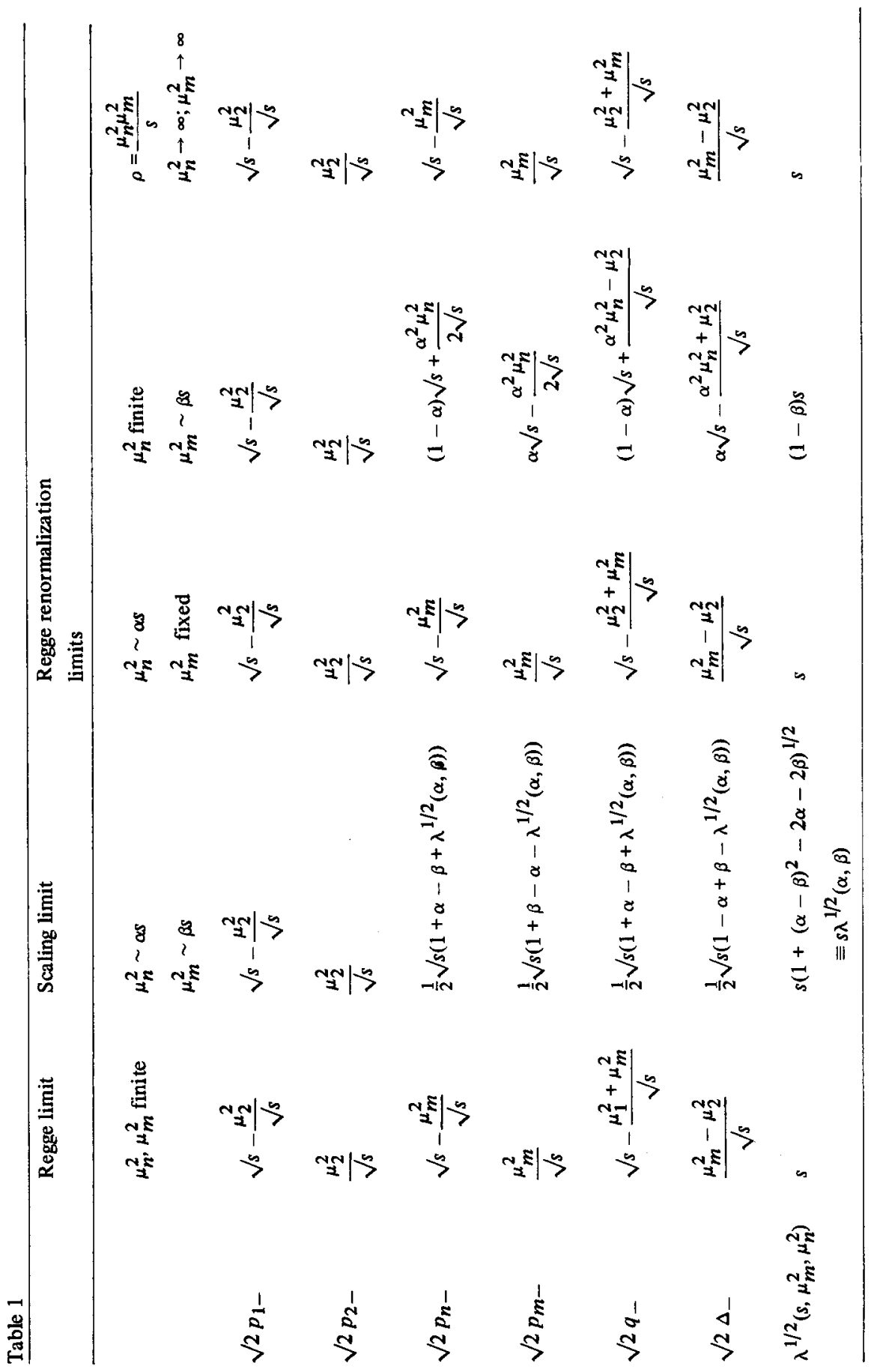


of the calculation by presenting the details for two terms.

Referring to table 1 , we note that $p_{2-}$ and $p_{m-}$ both tend to zero as $1 / \sqrt{ } s$. This, in turn, implies that the dominant contribution to each of the integrals in eq. (21) comes for every gluon exchange denominator becoming $\mathrm{O}\left(s^{-1}\right)$. For example, in the first term, we face $\left(v p_{2}-u p_{m}\right)^{2} \approx\left(v \mu_{2}^{2}-u \mu_{m}^{2}\right)^{2} / s$. The arguments of $\phi_{1}$ and $\phi_{n}$ tend to 1 , i.e., the quark emitted by meson 1 and absorbed by meson $n$ carries all the momentum of the mesons. Recalling that

$$
\phi_{i}^{\mathrm{a} \overline{\mathrm{b}}}(x) \rightarrow k_{i}^{\mathrm{a} \overline{\mathrm{b}}}(1-x)^{\beta_{\mathrm{b}}} \quad \text { as } x \rightarrow 1,
$$

the asymptotic behavior of the first integral is

$$
\begin{aligned}
& \frac{\mu_{2}^{2} \mu_{m}^{2}}{s} s \iint \mathrm{d} u \mathrm{~d} v k_{1}^{\mathrm{a} \overline{\mathrm{b}}} \frac{\left(\frac{\mu_{m}^{2}-v \mu_{2}^{2}}{s}\right)^{\beta_{\mathrm{b}}}-\left(\frac{(1-u) \mu_{m}^{2}}{s}\right)^{\beta_{\mathrm{b}}}}{\left(v \mu_{2}^{2}-u \mu_{m}^{2}\right)^{2}} \\
& \quad \times k_{n}^{\mathrm{a} \overline{\mathrm{d}}}\left(\frac{(1-v) \mu_{2}^{2}}{s}\right)^{\beta_{\mathrm{d}}} \phi_{2}(v) \phi_{m}(u) \\
& =s^{-\beta_{\mathrm{b}}-\beta_{\mathrm{d}}}\left\{k_{1}^{\mathrm{a} \overline{\mathrm{b}}} k_{n}^{\mathrm{a} \overline{\mathrm{d}}}\right\}\left\{\mu_{2}^{2} \mu_{m}^{2}\right\} \iint \mathrm{d} u \mathrm{~d} v \phi_{2}(v) \phi_{m}(u) \\
& \quad \times \frac{\left[\left(\mu_{m}^{2}-v \mu_{2}^{2}\right)^{\beta_{\mathrm{b}}}-\left((1-u) \mu_{m}^{2}\right)^{\beta_{\mathrm{b}}}\right]}{\left(v \mu_{2}^{2}-u \mu_{m}^{2}\right)^{2}}\left((1-v) \mu_{2}^{2}\right)^{\beta_{\mathrm{d}}} .
\end{aligned}
$$

Note that the power is, as expected, related to the (wee) quarks $b$ and $d$ exchanged between mesons 1 and $m$ and between mesons 2 and $n$, respectively. Notice that the dependence of the coefficient of the power (Regge residue) on mesons 1 and 2 is entirely given by the factors $k_{1}^{\mathrm{a} \overline{\mathrm{b}}} k_{n}^{\mathrm{a} \overline{\mathrm{d}}}$ in the first curly bracket.

The analysis of the third and fourth terms is slightly more complicated. Consider, e.g., the third integral. Because $p_{2}$ and $p_{m}$ tend to zero, the dominant regions of integration are $x$ and $y$ near 1 . To this end, let

$$
\begin{aligned}
& q(1-x) \equiv p_{m} \xi \\
& q(1-y) \equiv p_{2} \eta .
\end{aligned}
$$

Once again, the arguments of $\phi_{1}$ and $\phi_{n}$ tend to one. Noting also that $U \approx-s$, we must use the scaling relation for Green functions,

$$
G\left(1-\frac{\alpha}{s}, 1-\frac{\beta}{s} ; s\right) \underset{s \rightarrow \infty}{\longrightarrow} g(\alpha, \beta) .
$$

Then the asymptotic behavior of the third term is

$$
s^{-\beta_{\mathrm{b}}-\beta_{\mathrm{d}}}\left\{k_{1}^{\mathrm{a} \overrightarrow{\mathrm{b}}} k_{n}^{\mathrm{a} \overline{\mathrm{d}}}\right\}\left\{\mu_{2}^{2 \beta_{\mathrm{d}}} \mu_{m}^{2 \beta_{\mathrm{b}}} \iiint \int \mathrm{d} u \mathrm{~d} v \mathrm{~d} \xi \mathrm{d} \eta \phi_{m}(u) \phi_{2}(v)\right.
$$




$$
\left.\times g_{-}\left(\xi \mu_{m}^{2}, \eta \mu_{2}^{2}\right)\left[\frac{(1+\eta)^{\beta_{\mathrm{d}}}-(1-v)^{\beta_{\mathrm{d}}}}{(\eta+v)^{2}}\right]\left[\frac{(1+\xi)^{\beta_{\mathrm{d}}}-(1-u)^{\beta_{\mathrm{b}}}}{(\xi+u)^{2}}\right]\right\}
$$

$g(\alpha, \beta), g_{-}(\alpha, \beta)$ are the scaling functions defined in eqs. (A.10), (A.11).

So it goes with each integral. In every term the dependence on mesons 1 and $n$ of the Regge residue is expressed by $k_{1}^{\mathrm{ab}} k_{n}^{\mathrm{ad}}$. Thus, not only does each term exhibit $t$-channel factorization but so does their sum. By parity invariance, the full sum must be proportional to

$$
s^{-\beta_{\mathrm{b}}-\beta_{\mathrm{d}}} k_{1}^{\mathrm{a} \overline{\mathrm{b}}} k_{n}^{\mathrm{a} \overline{\mathrm{d}}} k_{2}^{\mathrm{c} \overline{\mathrm{d}}} k_{m}^{\mathrm{c} \overline{\mathrm{b}}}
$$

(At this point we must admit that we have not succeeded in showing that the proportionality constant is non-zero.) As is frequently the case in the $A_{-}=0$ gauge, parity relations are not trivial and we have not attempted to demonstrate this final form directly from the integrals above. At this level in the $1 / N_{\mathbf{c}}$ expansion we have etablished that one can build pairs of signaturized amplitudes, looking exactly like strongly exchange-degenerate Regge exchanges. If eq. (27) is indeed an equality, one has also shown that the leading Regge exchanges have $c \tau=+$ (the equivalent of charge conjugation times signature) if eq. (27) is just a proportionality relation and a different one holds for the $(s, t)$ diagram one can construct also $c \tau=-1$ exchanges *.

One may be interested in the non-leading behavior as well, but this is difficult to discuss without knowing precisely the rate for which the wave functions approach $k(1-x)^{\beta}$ as $x \rightarrow 1$. Naive consideration of the individual integrals suggests the presence of negative integer powers, but whether these powers actually survive or are cancelled is an open question.

The contribution to $\operatorname{Im} P$ of this region of phase space is $s^{2 \alpha-1}$, reminiscent of the AFS cut [16]. Whether the sum over states $n, m$ leads to a non-zero coefficient is an especially interesting question also left unanswered here. Later, we shall return to Regge renormalization effects, but after this warm-up, we turn to the primary subject of this paper.

\subsubsection{The "pomeron" limit}

As discussed earlier in sect. 2, we want to consider the region of phase space where both $\mu_{m}^{2}$ and $\mu_{n}^{2}$ are of order $s$, say $\mu_{n}^{2}=\alpha s \mu_{m}^{2}=\beta s$. The parameters $\alpha$ and $\beta$ are related to the momentum fractions of the q $\overline{\mathrm{q}}$ pairs in mesons 1 and 2 according to $\alpha=x_{1}\left(1-x_{2}\right)$ and $\beta=x_{2}\left(1-x_{1}\right)$. Accordingly, the allowed values of $\alpha$ and $\beta$ are most simply specified by noting that $x_{1}, x_{2} \in(0,1)$. The kinematics of this limit is given by table 1 , where we see that a principal difference from the Regge limit is that $p_{m-}$ is $\mathrm{O}(\sqrt{ } s)$ instead of $\mathrm{O}(1 / \sqrt{s})$. Consider the first integral in eq. (21). The dominant region comes from $u \approx 0$; to this end, we define $u p_{m} \equiv \eta p_{2}$. Now, even though the arguments of $\phi_{n}$ and $\phi_{m}$ tend to end points, both wave functions,

* We wish to thank Y. Eylon for discussions related to this point. 
in fact, scale [(A.10) and (A.11)]:

$$
\begin{aligned}
& \phi_{m}(u)=\phi_{m}\left(\frac{\eta p_{2}}{p_{m}}\right) \approx \phi^{\mathrm{c}}\left(\frac{\mu_{m}^{2} \eta p_{2}}{p_{m}}\right), \\
& \phi_{n}\left(\frac{q+v p_{2}}{p_{n}}\right)=\phi_{n}\left(1-\frac{p_{2}}{p_{n}}(1-v)\right) \simeq(-)^{n} \phi^{\mathrm{d}}\left(\frac{\mu_{n}^{2} p_{2}}{p_{n}}(1-v)\right) .
\end{aligned}
$$

Now consider meson 1 . The arguments of both terms tend to the same fixed value, viz. $x_{1} \equiv p_{m} / p_{1}$ (note $0<x_{1}<1$ ); hence, unlike the Regge limit, the terms cancel. Therefore, provided the remaining integrations are finite, the scaling asymptotic behavior has decoupled. Altogether we get

$$
\begin{aligned}
& \int_{0}^{\infty} \mathrm{d} \eta \phi^{\mathrm{c}}\left(\frac{\mu_{m}^{2} p_{2}}{p_{m}} \eta\right) \int_{0}^{1} \mathrm{~d} v \frac{\left[\phi_{1}\left(p_{m} / p_{1}\right)-\phi_{1}\left(p_{m} / p_{1}\right)\right]}{(v-\eta)^{2}} \\
& \quad \times(-)^{n} \phi^{\mathrm{c}}\left(\frac{\mu_{n}^{2} p_{2}}{p_{n}}(1-v)\right) \phi_{2}(v) .
\end{aligned}
$$

The integral over $\eta$ is seen to converge at $\infty$. Convergence at $\eta=0$ is somewhat less obvious. Letting $v=\eta z$, we find that the integrand behaves as $\eta^{2 \beta_{c}-1}$ and so is integrable at $\eta=0$. The next term in the expansion comes from

$$
(-)^{n} \phi_{1}^{\prime}\left(\frac{p_{m}}{p_{1}}\right)\left(\frac{p_{2}}{p_{1}}\right) \int_{0}^{\infty} \mathrm{d} \eta \phi^{\mathrm{c}}\left(\frac{\mu_{m}^{2} p_{2}}{p_{m}} \eta\right) \int_{0}^{1} \mathrm{~d} v \frac{\phi^{\mathrm{c}}\left(\frac{\mu_{n}^{2} p_{2}}{p_{n}}(1-v)\right) \phi_{2}(v)}{v-\eta}
$$

The integral over $\eta$ is of the form

$$
\int^{\infty} \frac{\mathrm{d} z \sin z}{z}
$$

at the upper limit and, hence, is convergent. Since $p_{2} / p_{1}=\mathrm{O}\left(s^{-1}\right)$, we find the asymptotic behavior to be nominally $s^{-1}$. (We say nominally since we have not proved the coefficient is non-zero.)

All the other integrals may be treated in a similar fashion with the same sort of cancellation occurring by pairs. Is there a simple physical reason for the cancellation? Noting again the vanishing of the gluon propagator, we infer that the cancellation reflects the sum of a quark's light-like color charge $Q_{-}$with an antiquark's opposite color charge.

Finally we note that we have also calculated the contribution to the unitarity sum coming from high mass production by the $(s, t)$ diagram mechanism, in that case even in each time-ordering no new singularity unrelated to quark parameters is built up.

We turn next to regions of phase space responsible for Regge intercept and vertex renormalizations. 


\subsubsection{Regge renormalizations}

(a) Vertex renormalization. Consider the region in which $\mu_{m}^{2}$ becomes large, say $\mu_{m}^{2}=\beta$ s, but $\mu_{n}^{2}$ stays finite (fig. $2 \mathrm{a}$ ). We anticipate that this should lead to a vertex renormalization of the $(\mathrm{d} \overline{\mathrm{d}})$ trajectory, thereby breaking strong exchange degeneracy. Proceeding in a manner analogous to the pomeron discussion above, we find a cancellation occurs in the first three integrals, so that these do not cause a breaking in the leading trajectory. However, the fourth integral appears to give a Regge power, which can be seen as follows: Referring to the kinematics in table 1 , it is easy to see that the leading asymptotic behavior of the amplitude, eq. (21), comes for $y \simeq 1$ and $v$ fixed. To this end, let $y \Delta_{-}=\eta p_{2-}$. Then one finds

$$
\begin{aligned}
& \approx \\
& \approx \frac{\Delta}{p_{n}} \iiint \int \mathrm{d} x \mathrm{~d} y \mathrm{~d} u \mathrm{~d} v G\left(x, p_{2} \eta / p_{n} ;-\beta\right) \phi_{n}(u) \phi_{2}(v) \\
& \quad \times\left[\frac{\phi^{\mathrm{c}}\left(\mu_{2}^{2}(1+\eta)\right)-\phi^{\mathrm{c}}\left(\mu_{2}^{2} v\right)}{(\eta+1-v)^{2}}\right]_{1}^{\phi_{1}\left(\frac{p_{n}+x p_{m}}{p_{1}}\right)-\phi_{1}\left(\frac{u p_{n}}{p_{1}}\right)} \\
& \left(\frac{p_{m}}{p_{n}} x+1-u\right)^{2}
\end{aligned}
$$

Inserting the definition of the Green function $G$ and using $\phi_{l}(x) \rightarrow k_{l}^{\mathrm{db}} x^{\beta_{\mathrm{d}}}$ as $x \rightarrow 0$, we can write

$$
\begin{aligned}
& \frac{p_{m}}{p_{n}} \sum_{l} \frac{k_{l}^{\mathrm{d} \overline{\mathrm{b}}}}{\mu_{l}^{2}+\beta} \iiint \int \mathrm{d} x \mathrm{~d} y \mathrm{~d} u \mathrm{~d} v\left(\frac{p_{2} \eta}{p_{m}}\right)^{-\beta_{\mathrm{d}}} \phi_{2}(v)\left[\frac{\phi^{\mathrm{c}}\left(\mu_{2}^{2}(1+\eta)\right)-\phi^{\mathrm{c}}\left(\mu_{2}^{2} v\right)}{(\eta+1-v)^{2}}\right] \\
& \times \phi_{l}(x) \phi_{n}(u) \frac{\phi_{1}\left(\frac{p_{n}+x p_{m}}{p_{1}}\right)-\phi_{1}\left(\frac{u p_{n}}{p_{1}}\right)}{\left(\frac{p_{m}}{p_{n}} x+1-u\right)^{2}}
\end{aligned}
$$

Since $p_{2-} / p_{m-} \propto s^{-1}$, we see that this is proportional to $s^{-\beta_{\mathrm{d}}}$. When squared, this gives a contribution to $\operatorname{Im} P \propto s^{-2 \beta_{\mathrm{d}}}$, which we interpret as a renormalization of the

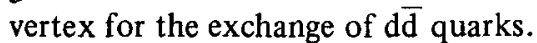

If we had instead let $\mu_{n}^{2}=\alpha s$ and $\mu_{m}^{2}$ finite, we expect to find a contribution to Im $P \propto s^{-2 \beta_{\mathrm{b}}}$, which would renormalize the vertex for (b $\left.\bar{b}\right)$ exchange. It is a simple (by now) calculation to show that this is indeed the case. The lack of manifest parity invariance reveals itself in an amusing way: Whereas in the previous calculation, only one of the form integrals survived in the limit, in this case we find all four terms in the amplitude eq. (21) yield the same power $s^{-\beta_{\mathrm{d}}}$. We have no explanation for this and, in fact, have not been demonstrated that the vertex given, e.g., by eq. (31) is non-zero. We also have not been able to show that the renormalized singularity factorizes.

(b) Intercept renormalization. Our experience with multiperipheral or dual 
models leads us to suspect that an intercept renormalization may come from the region of phase space for which both $\mu_{n}^{2}$ and $\mu_{m}^{2}$ increase as $s$ grows, but such that $\rho=\mu_{n}^{2} \mu_{m}^{2} / s$ remaining finite (fig. $2 \mathrm{~b}$ ). The rather complicated kinematics of this limit are summarized in table 1 . Proceeding to analyze each integral, we again find that the first three cancel but the fourth survives to give the result anticipated. For simplicity, one may choose the symmetric case where $\mu_{n}^{2}$ and $\mu_{m}^{2}$ are $\mathrm{O}(\sqrt{ } s)$. Again, we set $y \Delta_{-} \equiv \eta p_{2}$ - but unlike the vertex renormalization above, we have $\Delta_{-} / p_{n-} \rightarrow 0$, so we must let $u \rightarrow 1$. Thus, we set $(1-u) p_{n_{-}} \equiv \xi \Delta_{-}$. One can easily check that, in this limit, $T=\Delta_{\mu}^{2} \rightarrow-\rho$. Not only does $\phi_{m}$ scale, but also $\phi_{n}$ scales, and we find

$$
\begin{aligned}
& (-)^{n} k_{1}^{\mathrm{a} \overline{\mathrm{b}}} \sum_{l} \frac{k_{l}^{\mathrm{d} \overline{\mathrm{b}}}}{\mu_{l}^{2}+\rho} \iiint \int \mathrm{d} x \mathrm{~d} y \mathrm{~d} \xi \mathrm{d} v\left(\frac{p_{2} \eta}{p_{m}}\right)^{\beta_{\mathrm{d}}} \phi_{2}(v)\left[\frac{\phi^{\mathrm{c}}\left(\mu_{2}^{2}(1+\eta)\right)-\phi^{\mathrm{c}}\left(\mu_{2} v\right)}{(\eta+1-v)^{2}}\right] \\
& \times \phi_{l}(x) \phi^{\mathrm{d}}(\rho \xi)\left[\frac{\left((1-x) p_{m} / p_{1}\right)^{\beta_{\mathrm{b}}}-\left((1+\xi) p_{m} / p_{1}\right)^{\beta_{\mathrm{b}}}}{(x+\xi)^{2}}\right]
\end{aligned}
$$

This is proportional to

$$
\left(\frac{p_{2}}{p_{m}}\right)^{\beta_{\mathrm{d}}}\left(\frac{p_{m}}{p_{1}}\right)^{\beta_{\mathrm{b}}} \propto\left(\mu_{m}^{2}\right)^{-\beta_{\mathrm{d}}}\left(\mu_{n}^{2}\right)^{-\beta_{\mathrm{b}}},
$$

which is the result sought for.

\section{Summary}

In this paper, we have shown that, unlike the dual resonance model, the cylinder graph in (QCD) ${ }_{2}$ does not lead to a new singularity which one might want to identify with a bare pomeron. It might happen that, inclusion of quark-antiquark pairs (the "sea") in higher orders could generate a new singularity. It seems likely that the absence of this new singularity to order $1 / N^{2}$ is related to the absence of gluonic degrees of freedom and gluonic bound states ("glueballs") in two spacetime dimensions. If so, an analogous calculation in (QCD) ${ }_{4}$ ought to yield a bare pomeron. The attempts to derive the bare pomeron in (QCD) are at a preliminary stage. Low [17] and Nussinov [18] have introduced schemes in which the pomeron indeed appears as a singularity at one. Gunion and Soper [19] have summed a certain class of diagrams in QCD to obtain the Low-Nussinov type results, they also obtain a decoupling of the bare pomeron at $D=2$ for reasons similar to those we address *. Recently Veneziano [20] has presented a unified approach to QCD and the topological expansion. The bare pomeron he discusses coincides with the one we had set out to calculate in this paper in $(\mathrm{QCD})_{2}$, it seems to us that the bare

* While their results are much nearer to the desired ones, we think that the interest in our work $[8,9]$ is that we derive the properties of high energy diffractive scattering in a well-defined field theory which is both asymptotically free and confining. 
pomeron he describes in $(\mathrm{QCD})_{4}$ is the one whose properties should be pursued. However, in the context of the topological expansion, it has been conjectured [21] that no new singularity results from the cylinder graph, in which case, the situation would more nearly resemble our two-dimensional calculation.

Two technical improvements on our method seem feasible and desirable. It would be a considerable increase in efficiency if the asymptotic limits could be extracted without decomposing the covariant amplitudes into a sum of "time" -ordered amplitudes. We have done this for the virtual Compton amplitude [22] and believe it is possible in general by introducing Sudakov variables.

Secondly, it would be useful if parity invariance were made manifest, even at the expense of Lorentz invariance. To this end, it would be useful to solve the theory in the $A_{1}=0$ (Coulomb) gauge. In this gauge, parity is manifest and the language of Feynman's discussion [5] should be transcribable almost verbatim. As Feynman showed, the infinite momentum frame may restore simplicity even though Lorentz invariance is not manifest. However, in this regard, we must note that there are puzzling questions of consistency in axial gauges [23].

Finally, it may be of considerable theoretical interest if one could solve the model in a covariant gauge or any gauge in which the self-coupling of gluons is non-zero. One could even hope to get some insight into the four-dimensional confinement problem.

We especially wish to acknowledge S. Nussinov, We also thank W. Bardeen, R. Brower, G.F. Chew, J. Ellis, Y. Eylon, J. Gunion, A. Mueller and G. Veneziano for stimulating discussions and correspondence.

\section{Appendix A}

\section{Review of Feynman rules and previous results}

The Feynman rules and results have been summarized elsewhere [1]. We recall that, in the $A_{-}=0$ gauge with light-cone-quantization, the Coulomb gluon exchange may be characterized by a "propagator"

$$
\frac{1}{k_{-}^{2}} \equiv \frac{1}{2}\left[\frac{1}{\left(k_{-}+i \epsilon\right)^{2}}+\frac{1}{\left(k_{-}-i \epsilon\right)^{2}}\right] \text {. }
$$

The exact, dressed quark propagator is

$$
S_{\mathrm{R}}(p)=\frac{p_{-} \gamma_{+}+\left(m_{0}^{2} / 2 p_{-}\right) \gamma_{-}+m_{0}}{p^{2}-m^{2}}
$$

The renormalized mass is related to the bare quark mass $m_{0}$ by $m^{2}=m_{0}^{2}-g^{2} N / \pi$. We take units where $g^{2} N / \pi=1$. As before, because $S(p)$ always enters a calculation sandwiched between $\gamma_{-}$matrices, it is useful to define $\gamma_{-} S_{\mathrm{R}}(p) \gamma_{-}=2 S(p) \gamma_{-}$

$$
S(p)=\left[p_{+}-\frac{m^{2}}{2 p_{-}}\right]^{-1} .
$$


The proper vertex for the meson-quark-antiquark is denoted by $\Gamma_{n}(p, r)$, where $r(p)$ is the momentum of the meson (quark). In fact, in the light-cone gauge, this has the form

$$
\Gamma_{n}(p, r)=\frac{1}{r_{-}} \Gamma_{n}(x),
$$

where $x \equiv p_{-} / r_{-}$. For $0 \leqslant x \leqslant 1$, this is simply related to bound state wave function $\phi_{n}(x)$ by

$$
\Gamma_{n}(x)=-\left[\mu_{n}^{2}-\frac{m_{\mathrm{a}}^{2}-1}{x}-\frac{m_{\mathrm{b}}^{2}-1}{1-x}\right] \phi_{n}(x) .
$$

The proper vertex is defined for all $x$ by the integral

$$
\Gamma_{n}(x)=\int_{0}^{1} \frac{\mathrm{d} y \phi_{n}(y)}{(y-x)^{2}}
$$

where, for $0 \leqslant x \leqslant 1$, we take the principal value prescription. In this region, combining these two equations gives 't Hooft's bound state equation. The $T$-matrix for quark-antiquark scattering in the color singlet channel is given by

$$
T\left(k_{-}, l_{-} ; r\right)=\frac{\pi}{N} \frac{1}{\left(k_{-}-l_{-}\right)^{2}}-\frac{\pi}{N r_{-}^{2}} \sum_{n} \frac{\Gamma_{n}(x) \Gamma_{n}(y)}{r^{2}-\mu_{n}^{2}},
$$

where $x=k_{-} / r_{-}, y=l_{-} / r_{-}$are the momentum ratios for the initial and final quarks. A number of other relations are useful: Defining, as before, the Green function

$$
G\left(x, y ; r^{2}\right)=\sum_{n} \frac{\phi_{n}(x) \phi_{n}(y)}{r^{2}-\mu_{n}^{2}}
$$

we may also write the $T$-matrix as

$$
T\left(k_{-}, l_{-} ; r\right)=\frac{\pi}{N r_{-}^{2}}\left\{\frac{1}{(x-y)^{2}}-\int_{0}^{1} \int_{0}^{1} \frac{\mathrm{d} u \mathrm{~d} v G\left(u, v ; r^{2}\right)}{(u-x)^{2}(v-y)^{2}}\right\}
$$

The Green function $G\left(x, y, r^{2}\right)$ obeys the scaling relation $(25)$ where $g(\alpha, \beta)$ is given by

$$
g(\alpha, \beta)=\frac{1}{\pi^{2}} \int_{0}^{\infty} \frac{\mathrm{d} \lambda}{\lambda-1+i \epsilon} \phi^{\mathrm{b}}(\alpha \lambda) \phi^{\mathrm{d}}(\beta \lambda) .
$$

In eq. (25) we have defined $g(\alpha, \beta)$ for a fixed real ratio $\alpha / \beta$ as an analytic function of $\alpha$ with a cut for positive, real $\alpha$. In particular we define

$$
g_{-}(\alpha, \beta)=g(-\alpha,-\beta) \text {. }
$$

Finally in the course of calculations we rely on some scaling properties of the 
wave function, i.e.

$$
\lim _{n \rightarrow \infty} \phi_{n}^{\mathrm{ab}}\left(\frac{\xi}{\mu_{n}^{2}}\right)=\phi^{\mathrm{a}}(\xi),
$$

where for $\xi>0$ the scaling function is the solution of the equation

$$
\left(1-\frac{m_{\mathrm{a}}^{2}}{\xi}\right) \phi^{\mathrm{a}}(\xi)=\int_{0}^{\infty} \frac{\mathrm{d} \eta \phi^{\mathrm{a}}(y)}{(\eta-\xi)^{2}} .
$$

\section{References}

[1] H.D. Politzer, Phys. Reports 14 (1974) 130.

[2] A. De Rujula, H. Georgi, H.D. Politzer, Trouble with $\xi$-scaling?, Harvard preprint HUTP-76/A179, and references therein.

[3] G. 't Hooft, Nucl. Phys. B75 (1974) 461; Gauge theory for strong interactions, ITP Utrecht, 1975, lectures given at Erice and Copenhagen summer Schools.

[4] C.G. Callan, N. Coote and D.J. Gross, Phys. Rev. D13 (1976) 1649; M.B. Einhorn, Phys. Rev. D14 (1976) 3451.

[5] R.P. Feynman, Photon-hadron interactions (Benjamin, Reading, 1972); P.V. Landshoff and J.C. Polkinghorne, Phys. Reports 5 (1972) 1.

[6] M.B. Einhorn, Failure of naive parton models in inclusive electron-positron annihilations, Phys. Rev., to be published;

J. Kipfganz and M.G. Schmidt, Nucl. Phys. B125 (1977) 323.

[7] G. 't Hooft, Nucl. Phys. B72 (1974) 461;

G. Veneziano, Nucl. Phys. B117 (1976) 519.

[8] R.C. Brower, J. Ellis, M.G. Schmidt and J.H. Weis, Ref. Th. 2233-CERN.

[9] M.B. Einhorn, S. Nussinov and E. Rabinovici, University of Michigan Preprint, UM HE 76-39 (1976).

[10] M.B. Einhorn, in preparation.

[11] J. Ellis, private communication.

[12] I. Bars, Phys. Rev. Letters 36 (1976) 1521.

[13] S.-H. Tye, Phys. Rev. 13D (1976) 3416.

[14] Dual Theory, ed. M. Jacob, (North-Holland, Amsterdam, 1974).

[15] See Einhorn, ref. [4].

[16] D. Amati, S. Fubini and A. Stangellini, Nuovo Cimento 26 (1962) 896.

[17] F.E. Low, Phys. Rev. D12 (1975) 163.

[18] S. Nussinov, Phys. Rev. Letters 34 (1975) 1286; Phys. Rev. D14 (1976) 246.

[19] J.F. Gunion and D.E. Soper, U.C. Davis preprint.

[20] G. Veneziano, Nucl. Phys. B117 (1976) 519.

[21] G.F. Chew and C. Rosenzweig, Phys. Letters 58B (1975) 93.

[22] M.B. Einhorn, unpublished.

[23] Y. Frishman, C. Sachrajda, H. Abarbanel and R. Blanckenbecker, SLAC PUB-1808 (1976);

A.J. Hanson, R.D. Peccei and M.K. Prasad, Nucl. Phys. B1 21 (1977) 477;

A.J. Hanson and M.K. Prasad, LBL-6117;

N.K. Pak and H.C. Tze, SLAC-PUB-1792 (1976). 